\title{
Clustering based Routing Protocols in Vehicular Ad-hoc Networks: A Review
}

Sanjeev Punia and Rajeev Kumar Patial

Department of Electronics and Communication, Lovely Professional University, Phagwara - 144411, Punjab , India; sanjeevpunia1914@gmail.com, rajeev.kumar@|pu.co.in

\begin{abstract}
Vehicular Ad Hoc Network (VANET) is subclass of Mobile Ad Hoc Network (MANET) which has become active area for research, standardization, and development because it has tremendous potential to improve vehicle and road safety, traffic efficiency. There are lot of challenges in VANET to route the packet to destination because of high mobility involved in the network. keeping the constraint of mobility in view, this paper provides a review of various routing techniques based on clustering algorithm. The main motive is to select a particular technique that exist in literature depending on the need of applications. Clustering in VANET is a control scheme to manage media access and make VANET as a global topology. Most algorithms and protocols are derived from Mobile Ad-hoc Network (MANET) which have some challenges and regarding issues. The architecture of VANETs with the application and constraints of routing protocols is explained in this paper. It searches the motivation behind the development of these clustering protocols. In this paper classification of various clustering routing protocols is done on the basis of following parameters i.e. research methodology, their advantages and disadvantage, various challenges and problems faced during the implementation of routing technique.
\end{abstract}

Keywords: Clustering, Routing Protocols, Vehicular Ad Hoc Network (VANET) , , V2V, V2I, ITS

\section{Introduction}

Due to the development of technology in Mobile Ad Hoc Network and in vehicles, a lot of momentum is gain in the field of Vehicular Ad Hoc Network (VANET).VANET have become a growing field of study for the researchers from different fields including Mechanics, Electronics, Networking etc. VANET represent a evolution of wireless ad hoc networks that enable inter vehicle communication and communication with infrastructure or roadside equipment. The term Intelligent Transportation System (ITS) refers to add information and communication technology to infrastructure and vehicles in order to improve the safety of road transportation and to reduce the traffic jams and fuel consumption of the vehicles Effective utilization of ITS can improve the driving comfort and it can also increase the safety and it helps to reduce the traffic jams. The communication in VANETs is carried out in two ways either from Vehicle-to-Vehicle or From Vehicle-to-Road side units installed on road sides or the nearby buildings. Communication in vehicles is possible through the On-Board-Unit (OBU) installed in the vehicles over a Dedicated Short Range communication (DSRC).

VANETs follow the IEEE 802.11p standards assigned to Wireless Ad Hoc Vehicular Environment (WAVE)]

In this paper, a detailed description of routing protocols which are used in clustering of vehicles is presented. Clustering based routing protocols are dependent on the position and size of clusters. The greatest advantage of Clustering based routing protocol in comparison to other routing protocols is that it helps to reduce the overhead involved in the network and delay of the network. It helps to increase the safety of the vehicles and it increases the packet delivery ratio.

\section{Overview of Vanets}

Vehicular Ad - hoc networks comprises of large no. of mobile nodes that are communicating with each other within a specified range of around $100-300 \mathrm{~m}$. The Federal Communication Commission (FCC) of United States

${ }^{*}$ Author for correspondence 
has allocated Dedicated Short Range Communication (DSRC) licensed spectrum of $75 \mathrm{MHz}$ with a bandwidth of $5.9 \mathrm{GHz}$ and protocol assigned to vehicular networks is IEEE 802.11p as discussed in section I. In Europe DSRC communication is carried out over a spectrum of $30 \mathrm{MHz}$ over $5.9 \mathrm{GHz}$ band which is used for many applications such as parking management, traffic telematics, transport management etc. As DSRC system of communication across Europe is not standardized, so it is not used in all the countries.

\subsection{VANET Architecture}

VANET architecture consists of a Road Side Unit (RSU) and an On-Board Unit (OBU) that is installed in the vehicles. The vehicles transmit messages from $\mathrm{OBU}$ to another OBU or from OBU to RSU and messages can be transceived from RSU to RSU as described in Figure 1.

If any vehicles transmits a message but there is no other vehicle in the specific range a certain vehicle, then the message is stored at the RSU and can be retrieved when any vehicle comes in the its rangef.

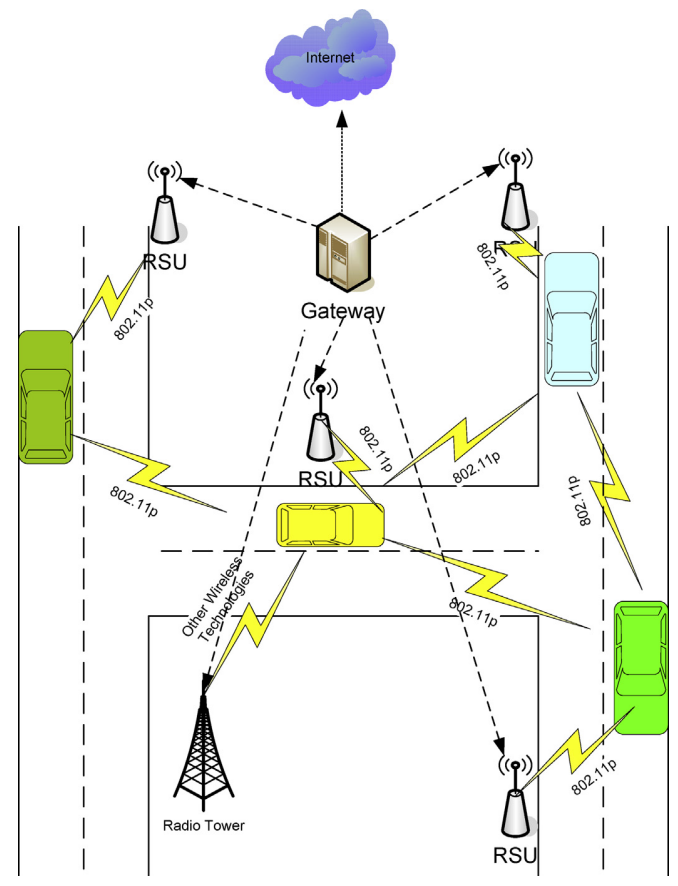

Figure 1. Generalized architecture in VANET.

\subsection{Communication Pattern in VANETs}

Various communication patterns with purpose, communication mechanism, direction, QoS is all described with an examplef
- Beaconing: Information is updated about the speed, position and the nearby vehicles among the nodes. The data packets are broadcasted through link layer over single-hop communication.

- Geobroadcast: Information about sudden occurrence of an even or an abnormality is broadcasted over a larger area in which sender attaches the determined location with message.

- Unicast Routing: Unicast transportation of messages in a specified direction. Multi-hop communication is more suitable for this communication.

- Advanced information Dissemination: Provides information to the vehicles that experiences a delay due to network partitioning. The messages with high priority are handled first when the bandwidth is available for a limited period.

- Information Aggregation: Communication overhead is reduced which in turn decreases the probability of collision and dropping of packets

\subsection{Applications of R'outing}

The Categories for routing application can be divided into three classes namely safety, transport efficiency and infotainment. Fast message dissemination and collision avoidance can be categorized under safety application. Route update and traffic monitoring are the major applications which are categorised under transport efficiency category. Other applications like asking for nearest petrol pump, nearest car parking location or room available in hotel or nearest service station which are not crucial but can increase comfort for people are categorized as infotainment application

Some more important applications of routing in VANET are:

\subsubsection{Alert Generations}

Vehicles which are moving towards intersection their information is gathered at RSU and analyses of the information takes place at RSU. Depending on the analysis if there is any chance of collision/accident, RSU will generate alert and inform approaching vehicles and they will take proactive measures to avoid such situation.

\subsubsection{Vehicle Maintenance}

Whenever there is a fault in the vehicle or when the driver has set a reminder for recall then the event driven warning 
are sent to vehicles. In case of any fault occurrence, messages are send by $\mathrm{OBU}$ to the infrastructure using V2I communication. The support centre replies back to the vehicle instructing the steps to be taken

\subsubsection{Security Services}

Critical safety conditions like traffic congestion, manmade or natural disaster, weather condition or hostile attack can occur in any highway or urban environment. In that kind of situation, multimedia message like video can be streamed to all the cars following behind it so they can be visually enlightened about the problem. These vehicles can make a better informed decision than if they simply got alarm text message

\section{Constraints and Challenges}

Due to the dynamic nature of VANETs, routing has various challenges and constraints with respect to management of Quality of Service (QoS) for various services:

- Rapid topology changes due to mobility.

- Variation in vehicle velocity and density on the road.

- Sparse distribution of vehicles in some geographical leads to loss in connectivity among vehicles resulting in declining performance of network

- Efficient clustering and selection of Cluster Head $(\mathrm{CH})$ based upon some predefined criteria

- Intrusion detection and security

\section{Clustering Based Routing}

In Clustering based routing schemes, one vehicle from each cluster is selected as cluster head $(\mathrm{CH})$ which main function is to manage other nodes in a cluster called as cluster members.

If any node which is in the range of two or more clusters, it is called as border node. There are number of techniques or protocols are proposed in literature for the selection of cluster head and how efficient routing is achieved through clustering. Some of the prominent protocols in this category are described in Figure 2.

Performance evaluation of routing protocols and cluster based flooding protocol for VANETs are proposed in LORA_CBR. There is a classification of nodes into $\mathrm{CHs}$, gateway or cluster member. There is one $\mathrm{CH}$ per cluster. If a node is connected to more than one cluster, it is termed as gateway. The information about the members and gateways is maintained by $\mathrm{CH}$. Routing of the packets from source to destination is done in greedy manner. In case the location of destination is not available, the Location Request (LREQ) packets are sent by the source. Only CH will disseminate the LREQ and Location Reply (LREP) messages.

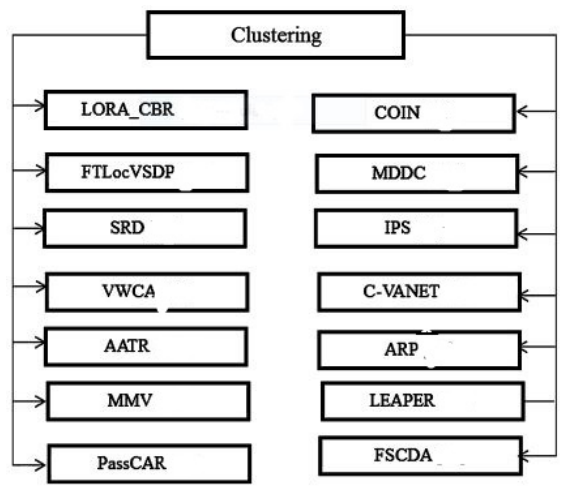

Figure 2. Classification of Clustering based routing.

An efficient fault tolerant service discovery protocol for VANETs is proposed in Due to faulty components between service provider and service requester there is decrease in dropped connections and service request satisfaction. This decrease in connections and service request satisfaction can be improved by fault tolerant techniques. In Fault Tolerant Location based Vehicular Service Discovery Protocol (FTLocVSDP) requester specifies the region of interest within the request and the protocol uses discovery of location based services.

Simple and Robust Dissemination (SRD) protocol is proposed in The SRD protocols combines an optimized broadcast suppression technique with store and carry forward mechanism. This Protocols operates in both sparse and dense network. The major problem in dense networks is broadcast storm and the SRD approach deals with it by using optimized broadcast suppression technique. For sparse networks where there are not many vehicles, the protocol uses store and carry forward communication technique.

A novel clustering algorithm naming vehicular clustering based on the weighted clustering algorithm (VWCA) is proposed in In VWCA algorithm number of parameters are taken into consideration like number of neighbour based on transmission range, velocity of the vehicle, direction of vehicle These above defined parameter helps to increase the stability and connectivity 
and reduces the overhead in the network. They also proposed adaptive allocation of transmission range (AATR) where transmission range is adaptively adjusted by hello Messages.

Passive clustering based routing protocol named PassCAR is proposed in In passive clustering, a cluster has one $\mathrm{CH}$ and multiple clusters can be connected through gateways. Pass CAR works in three phases namely route discovery, route establishment and data transmission. During the route discovery phase, suitable nodes are selected which become gateway and $\mathrm{CH}$. These nodes forward RREQ packets. For route establishment, the protocol uses multi-metric election strategy and considers the links reliability, stability and sustainability. Protocol quantifies the links based on parameters of node degree, expected transmission count and link lifetime. Once the route is discovered the destination node replies the RREP packet to the source node

Clustering for Open IVC Networks (COIN) algorithm is proposed in In this scheme, authors proposed that the $\mathrm{CH}$ is elected based on vehicular dynamics and the driver's intentions. Algorithm also takes into consideration the oscillatory nature of the inter vehicle distances. Simulations show that COIN produces relatively stable structure in VANETs. The overhead is also minimal and under tolerable limits.

A Multiagent Driven Dynamic Clustering (MDDC) of vehicles in VANETs is proposed in The various number of parameters have taken into consideration while proposing this algorithm like velocity and direction of vehicle, mobility pattern on a lane between two intersection. For rapid response for dynamic clustering this scheme comprises mobile and static agents. Cluster Head is selected among cluster members on the basis of parameters like average speed and connectivity degree. Cluster formation, $\mathrm{CH}$ selection and Cluster members selection time are simulation parameters for MMDC. The proposed scheme is compared to existing clustering schemes and it is found that MDDC performs better in all aspects

An Information Propagation Scheme (IPS) for VANETs is described in In this Scheme the concept of opportunistic flooding is followed. The Purpose of IPS is to warn the vehicles approaching to the accidental area to avoid danger or mistakes. The Author Focus on information propagation problems. For Example, What is the average speed of the road?, Wait time for a toll booth.
Such type of messages helps the vehicle to take decision earlier with caution. This Scheme is used in Vehicle to Vehicle distributed application

A Cooperative Communication Aware Link Scheduling for Cognitive VANET (C-VANET) is proposed in and have investigated the throughput maximization problem in C-VANET under multiple constraints such as

- Cognitive Radio (CR) devices' inherent single-ratio constraint

- The availability of licensed spectrum

- Transmission mode selection

- $\quad$ Link scheduling

A Adaptive Role Playing (ARP) strategy is proposed in ${ }^{15}$ in which data can be transferred in a reliable and faithful way. In ARP each node perform the basic task appointed to it so that in case if malfunctioning and misbehaving nodes are present in the network, the data packet can be relayed in reliable and faithful manner. Each individual node can act as either a relayer or a watchdog in a network. The number of watchdog in a cluster is dependent on a security threshold $\mathrm{K}$.

\section{Conclusion and Future Work}

From the Last few years, Vehicular Ad Hoc Network is the most emerging and new technology which is combination of networking and transportation and it is used in various application fields such as Rescue and surveillance operations, entertainment etc. For all these applications, there is a requirement of efficient routing techniques within the constraints such as Mobility and topological changes in the network. This paper provides a complete classification of various clustering based routing schemes with their relative advantages and disadvantages of each other. In future, we would implement one of the above defined schemes and compare its performance over the other schemes of its category.

\section{References}

1. Sultan S, Doori M, Bayatti A, Zedan H. A comprehensive survey on vehicular adhoc networks. J. Netw. Comput. Appl. January 2014; 37(1): 380-92.

2. Ami Dua, Neeraj Kumar, Seema Bawa. A systematic review on routing protocols for Vehicular Ad Hoc Networks.Vehicular Communications. January 2014; 1(1): 33-52. 
3. P1609.0/D5, Sep2012 - IEEE draf guide for Wireless Access in Vehic-ular Environments (WAVE) Architecture. http:// ieeexplore.ieee.org/servlet/opac? punumber $=6320593$. 2012;p.1-74.

4. Elmar Schoch, Frank Kargl, and Michael Weber. Communication patterns in VANETs. IEEE Communication Magazine. November 2008; 119-125.

5. Vishal Kumar, Shailendra Mishra, and Narottam Chand. Applications of VANETs: Present \& Future. Computer and Networks. February 2013; 5(18): 12-15.

6. Santos RA,, Edwards A, Edwards R, Seed L Performance evaluation of routing protocols in vehicular adhoc networks. Int.J. AdHoc Ubiq. Comput. November 2005; 1(1/2):80-91.

7. Abrougui K., Boukerche A, Ramadan H. Performance evaluation of an efficient fault tolerant service discovery protocol for vehicular networks. ,J.Netw. Com-put.Appl. 2012; 35(5):1424-35.

8. Schwartz RS. Barbosa RRR., Meratina N,.Heijenk G,. Scholten H A directional data dissemination protocol for vehicular environments. Comput.Commun. 2011;34(17): 2057-71.
9. Daeinabi A, Rahbar AGP, Khademzadeh A. VWCA:An efficient clustering algorithm in vehicular adhoc networks. J. Netw. Comput. Appl. January 2011; 34(1):207-22.

10. Wang SS, Lin YS. PassCAR: A passive clustering aided routing protocol for vehicular adhoc networks. Comput.Commun. January 2013; 36(2):170-9.

11. Blum J, Eskandarian A,. Hoffman L Mobility management in IVC networksin: Proceedings of IEEE Intelligent Vehicles Symposium, Columbus. Ohio,USA:June 2003.

12. Kakkasageri MS, Manvi SS. Multi agent driven dynamic clustering of vehicles in VANETs. J.Netw.Comput. Appl. 2012;35 (6):1771-80.

13. Little TDC.. Agarwal A, An information propagation scheme for VANETs, in: Proceedings of Intelligent Transportation Systems. September 2005.

14. Pan M,. Li P, Fang Y. Cooperative communication aware link scheduling for cognitive vehicularnetworks. IEEE J.Sel. AreasCommun. 2012; 30(4):760-8.

15. Li Z, Chigan C. LEAPER: a light weight reliable and faithful data packet relaying framework for VANETs. AdHoc Netw. 2011; 9(3): 418-42. 